\title{
Synthesis of New Heterocyclic Compounds Derived from Anthrone and Evaluation of Their Biological Activity
}

\author{
Nadia Adil Salih*
}

Date of acceptance 3/6/2008

\begin{abstract}
:
In this research, Schiff bases derived from the reaction of anthrone with different heterocyclic amines have been described. The resulted Schiff base compounds were reacted with various nucleophiles in order to obtain new heterocyclic derivatives. Chemical structures of all products were confirmed by IR, ${ }^{1} \mathrm{H}-,{ }^{13} \mathrm{C}-\mathrm{NMR}$ spectral data and elemental analysis. All synthesized compounds were in vitro tested against a standard strain of pathogenic microorganism including Gram +ve bacteria (Staphylococcus aureus), Gram -ve bacteria (Escherichia coli), and fungi (Candida albicans).
\end{abstract}

Keywords: Schiff base, Biological activity, Imidazole, 1,3,4-Thiadiazole

\section{Introduction:}

Schiff bases are used as substrates in the preparation of a number of industrial and biologically active compounds via closure, cycloaddition and replacement reactions. Moreover, Schiff bases are also known to have biological activities such as antimicrobial, antifungal, antitumor, and as herbicides[1]. Schiff bases have also been employed as ligands for complexation of metal ions[2]. On the industrial scale, they have a wide range of applications such as dyes and pigments[3].

Because of its synthetic utility and broad range of pharmacological activities, the benzimidazole nucleus is an important heterocyclic ring and its synthesis has received much attention. Benzimidazoles can be synthesized by a number of methods, usually involving formation of the N-C-N unit as the key step. One of the formally utilized general routes to benzimidazoles involves the reaction of aldehydes and ketones with $O$ - phenylenediamine. Although there are several routes leading to 2 -substituted benzimidazoles, a typical procedure involves heating $o$-phenylenediamine with a substituted carboxylic acid in the presence of mineral acid[4,5].2Substituted benzimidazoles with various types of biological activities, such as antibacterial[6], antiviral[7], antitumor[8] and anti-inflammatory[9], have been reported.

Schiff base of 4aminoantipyrine and its complexes have a variety of applications in biological, clinical, analytical and pharmacological areas[10]. Studies of a new kind of chemotherapeutic Schiff bases are attracting the attention of biochemists[11]. In addition, pyrazole derivatives have played a crucial part in the development of theory in heterocyclic chemistry and also used extensively in organic synthesis[12,13].

Heterocyclic compounds possessing 1,3,4-thiadiazole ring system show antifungal, bacteriostatic, anthelmintic[14] effect as well as

*Chemistry Department/ College of Science/ Al-Nahrain University/ Baghdad/ Iraq 
depression effect on central nervous system[15]. More recently, researchers reported 1,3,4-thiadiazole derivatives that exhibited analgesic and antiinflammatory activities[16].

\section{1,3-Thiazolidin-4-one}

derivatives possess anticonvulsant, hypnotic, anticancer properties and have been reported as novel inhibitors of the bacterial enzyme Mur B which is a precursor acting during the biosynthesis of peptidoglycan[17].

The incorporation of the imidazole nuclei is an important synthetic strategy in drug discovery. Many imidazoles have been prepared as pharmacological agents Azomycine, Clotrimazole, Miconazole, Ergothionine, Clonidine and Moxonidine[18]. One of the most important applications of imidazole derivatives is their used as reline material for treatment of denture stomatities[19].

These observations have encouraged me to synthesis some new products containing the above mentioned heterocyclic moiety hoping to obtain new compounds with potential biological activity.

\section{Experimental:}

\section{Instruments}

Melting points were determined on GallenKamp melting point apparatus and were uncorrected. The IR spectra of the compounds were recorded on Shimadzu FTIR-8300 spectrometer as $\mathrm{KBr}$ disc; results are given in $\mathrm{cm}^{-1}$. ${ }^{1} \mathrm{H}-\mathrm{NMR}$ and ${ }^{13} \mathrm{C}-\mathrm{NMR}$ spectra were recorded at 300 and 75 $\mathrm{MHz}$, respectively, in DMSO- $\mathrm{d}_{6}$ for all compounds on a Bruker AMX-400 NMR spectrometer. The chemical shifts are reported in part per million (ppm) downfield from internal tetramethylsilane (TMS) (chemical shift in $\delta$ values). Elemental analysis was run using a Perkin-Elmer RE 2400
CHNS analyzer. ${ }^{1} \mathrm{H}-,{ }^{13} \mathrm{C}-\mathrm{NMR}$ and elemental analysis were made at Chemistry Department, Georgia State University (Georgia, USA).

\section{Chemicals}

\section{Anthrone (10-anthracen-9-one)}

This material was supply from Fluka Chemical Company as synthetic material and used directly without further purification.

\section{Synthesis of 1',3'-dihydro-10H- spiro[anthracene-9,2'- benzimidazole] (1)}

A mixture of anthrone $(0.01 \mathrm{~mol}$, $1.94 \mathrm{~g})$ and $o$-phenylendiamine $(0.01 \mathrm{~mol}, 1.08 \mathrm{~g})$ in $20 \mathrm{ml}$ abs. ethanol was heated under reflux for $24 \mathrm{~h}$. Then the solvent was reduced to one third its volume under reduced pressure and then cooled. The solid that separated was recrystallized from chloroform.

\section{Synthesis of anthracen-9(10H)-one thiosemicarbazone (2)}

Thiosemicarbazide $(0.02 \mathrm{~mol}, \quad 0.91 \mathrm{~g})$ was added to $(0.02 \mathrm{~mol}, 3.88 \mathrm{~g})$ of anthrone dissolved in glacial acetic acid $(25 \mathrm{ml})$, the reaction mixture was refluxed for $10 \mathrm{~h}$. The solid that formed was separated by filtration and recrystallized from ethanol.

Synthesis of 2-(anthracen- 9(10H)ylidenehydrazono) thiazolidin -4-one (3)

To a mixture of compound $2(0.01 \mathrm{~mol}$, $2.99 \mathrm{~g}$ ) and potassium hydroxide $(0.01 \mathrm{~mol}, 0.56 \mathrm{~g})$ in $(20 \mathrm{ml})$ abs. ethanol, chloroacetic acid $(0.01 \mathrm{~mol}$, $0.95 \mathrm{~g}$ ) was added gradually. The reaction mixture was refluxed for $24 \mathrm{~h}$; the solid product was filtered off and recrystallized from acetone to obtain the desired product. 
Synthesis of 2-(anthracen-9(10H)ylidenehydrazono)- 5-bromophenyl2,3-dihydro-1H-thiazole (4)

A mixture of compound $2(0.01 \mathrm{~mol}$, $2.99 \mathrm{~g}$ ) and $p$-bromophenacyl bromide $(0.01 \mathrm{~mol}, 1.08 \mathrm{~g})$ in $(25 \mathrm{ml})$ abs. ethanol was refluxed for $24 \mathrm{~h}$; the solvent was reduced under reduced pressure and then cooled. The solid that separated was recrystallized from dichloromethane.

Synthesis of 4-(anthracen-9(10H)ylideneamino)- 1, 5- dimethyl- 2phenyl-1,2-dihydro-3H-pyrazol -3one (5)

A mixture of anthrone $(0.012 \mathrm{~mol}$, $2.33 \mathrm{~g}$ ), $15 \mathrm{ml}$ glacial acetic acid and 4aminoantipyrine $(0.012 \mathrm{~mol}, \quad 2.44 \mathrm{~g})$ was heated under reflux for $10 \mathrm{~h}$. The reaction mixture was filtered off and recrystallized from ethanol.

Synthesis of N-anthracen-9(10H)ylidenehistidine (6)

The same method described for synthesis of compound 5 but used Lhistidine $(0.012 \mathrm{~mol}, 1.86 \mathrm{~g})$ instead of 4-aminoantipyrine.

Synthesis of $\mathrm{N}$-anthracen $-9(10 \mathrm{H})$ ylidene-4-methylpyridine-2-amine(7) The same method described for synthesis of compound 5 but used 2amino-4-methyl pyridine $(0.012 \mathrm{~mol}$, $1.31 \mathrm{~g}$ ) instead of 4-aminoantipyrine.

Synthesis of 4-(anthracen-9(10H)ylideneamino)-1-methyl- 3- oxo -2phenyl-2,3-dihydro-1H-pyrazole- 5carboxylic acid (8)

$(0.05 \mathrm{~mol}, 17.65 \mathrm{~g})$ of compound $\mathbf{5}$ is added to a solution of $(0.05 \mathrm{~mol}, 7.90 \mathrm{~g})$ of potassium permanganate and $(0.05 \mathrm{~mol}, 5.30 \mathrm{~g})$ of sodium carbonate in $(25 \mathrm{ml})$ water and the mixture is heated under reflux until the color of the permanganate has disappeared (15h). The reaction mixture was filtered while still hot to get rid of the $\mathrm{MnO}_{2}$ precipitate. Remove any excess of $\mathrm{MnO}_{2}$ by addition of a little oxalic acid. The cooled filtrate is acidified with sulphuric acid (20\%), the carboxylic acid precipitate is filtered off, washed with a little cold water and used without further purification.

Synthesis of 4-(anthracen-9(10H)ylideneamino)-5- (1H-benzimidazol2-yl)-1-methyl-2-phenyl-1,2-dihydro3H-pyrazol-3-one (9)

To $(0.01 \mathrm{~mol}, 4.09 \mathrm{~g})$ of compound $\mathbf{8}$, a mixture of $(0.01 \mathrm{~mol}, 1.08 \mathrm{~g}) \quad o$ phenylenediamine and few drops of conc. hydrochloric acid in $(15 \mathrm{ml})$ abs. ethanol was added. Then the mixture was heated under reflux for $24 \mathrm{~h}$. After cooling, the crude product was filtered off and recrystallized from chloroform.

Synthesis of 5-[1-(anthracen-9(10H)ylideneamino) -2- (1H-imidazol- 5yl)ethyl]-1,3,4-thiadiazol- 2-amine (10)(20)

A mixture of compound $6(0.015 \mathrm{~mol}$, $4.97 \mathrm{~g}$ ) and an equivalent amount of thiosemicarbazide in $\mathrm{POCl}_{3}(10 \mathrm{ml})$ was refluxed in a water bath for 1012h. After evaporating under reduced pressure, a solid product was obtained. This was recrystallized from ethanol to afford the desired product.

Synthesis of 2-(anthracen-9(10H)ylideneamino)-4-carboxylic acidpyridine (11)

The same method described for synthesis of compound $\mathbf{8}$, but by using compound $7(0.05 \mathrm{~mol}, 14.20 \mathrm{~g})$ as starting.

Synthesis of ethyl 2-(anthracen9(10H)- ylideneamino) pyridine -4carboxylate (12)

A mixture of the acid $11(0.01 \mathrm{~mol}$, $3.14 \mathrm{~g})$, abs. ethanol $(10 \mathrm{ml})$, and few drops of conc. sulfuric acid was 
refluxed for $10 \mathrm{~h}$, the reaction mixture was cooled to room temperature and then in the refrigerator for $5 \mathrm{~h}$. The solid product was filtered off washed and recrystallized from ethanol.

Synthesis of 2-(anthracen-9(10H)ylideneamino)pyridine-4carbohydrazide (13)

A mixture of ester $12(0.012 \mathrm{~mol}, 3.91 \mathrm{~g})$ and $80 \%$ hydrazine hydrate $(0.02 \mathrm{~mol}$, $1.50 \mathrm{ml})$ was refluxed for $5 \mathrm{~h}$, then abs. ethanol $(15 \mathrm{ml})$ was added and refluxed for further $8 \mathrm{~h}$. The separated precipitate was filtered and washed with cold water.

Synthesis of 2-(4-(anthracen-9(10H)ylideneamino)-4-(1,6-dihydro-1,2,4triazin-5(2 H)-one)pyridine (14)

Compound $13(0.01 \mathrm{~mol}, 3.28 \mathrm{~g})$ and chloroacetamide $(0.01 \mathrm{~mol}, 0.93 \mathrm{~g})$ were mixed together in $(20 \mathrm{ml})$ abs. ethanol. The reaction mixture was refluxed for $24 \mathrm{~h}$, the solvent was reduced to one third its volume under reduced pressure. The crude product was obtained by filtration, washed with water and recrystallized from chloroform.

\section{Antibacterial and Antifungal studies:}

All newly synthesized compounds were tested for their in vitro growth inhibitory activity against a standard strain of pathogenic microorganism including Grampositive bacteria (Staphylococcus aureus), Gram-negative bacteria (Escherichia coli) and a yeast-like pathogenic fungus (Candida albicans). The primary screening was carried out using the agar disc-diffusion method using Müller-Hinton agar medium. Bacteria including Staphylococcus aureus and Escherichia coli were grown in nutrient broth at $37^{\circ} \mathrm{C}$ for 24h. Candida albicans was grown in malt broth at $37^{\circ} \mathrm{C}$ for $48 \mathrm{~h}$. Sterile filter paper disc $(5 \mathrm{~mm})$ were moistened with the compound solution in dimethylsulphoxide of specific concentration $(200 \mu \mathrm{g} / \mathrm{disc})$. The plates were incubated at $37^{\circ} \mathrm{C}$, and the diameter of the growth inhibition zones were measured after $24 \mathrm{~h}$ in case of bacteria and $48 \mathrm{~h}$ in case of Candida albicans. Antibiotic discs for Streptomycin were additionally tested as positive control.

\section{Results and Discussion:}

Chemists have reported on the chemical, structural and biological properties of Schiff bases. Schiff bases are characterized by the $-\mathrm{N}=\mathrm{CH}$ (imine) group which imports in elucidate the mechanism of transformation in biological system. In this study i reported the synthesis, characterization of some new Schiff bases and study their pharmacological activities. The synthesis pathway leading to compounds (1-4) is given in Scheme I:

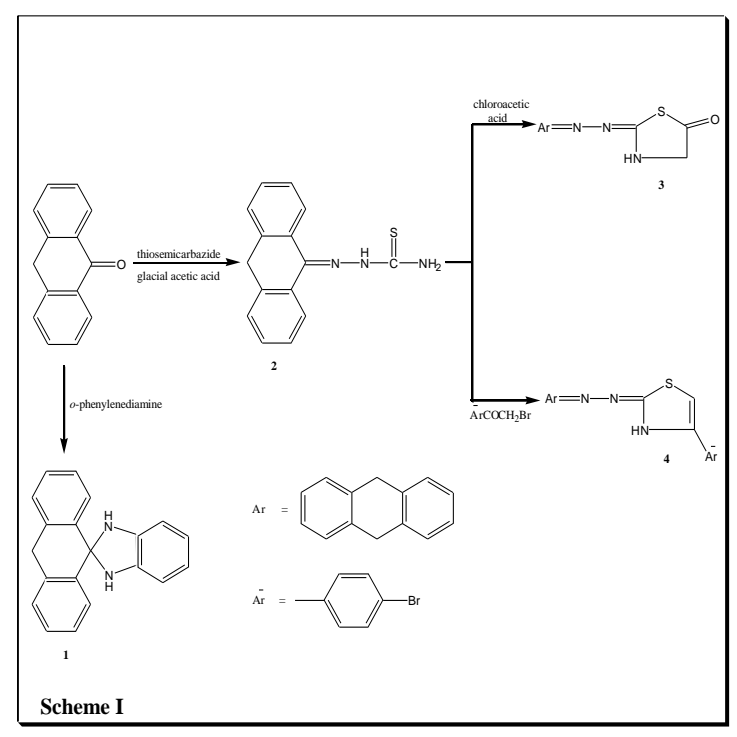


The structures of the new compounds 1-4 were assigned on the basis of their spectral data and elemental analysis. The IR spectra of all compounds were devoid of the band at about $1700 \mathrm{~cm}^{-1}$ due to the $\mathrm{C}=\mathrm{O}$ stretching vibration of the conjugated ketones[21]. ${ }^{1} \mathrm{H}-\mathrm{NMR}$ spectra of compounds 1-4 showed characteristic bands at $\delta 8.42-9.32$ belonging to $-\mathrm{NH}$ and $-\mathrm{NH}_{2}$ which are further characterized by disappearance with $\mathrm{D}_{2} \mathrm{O}$. Furthermore, ${ }^{13} \mathrm{C}-\mathrm{NMR}$ spectra showed multiple signals at $\delta 87.8$ $100.7,125.0-140.7$ for olefinic carbons and aromatic carbons, respectively. These signals gave a good support for ${ }^{1} \mathrm{H}-\mathrm{NMR}$ data.

The IR spectra of Schiff base compounds 5-7 showed characteristic $\mathrm{C}=\mathrm{N}$ bands at $1600-1630 \mathrm{~cm}^{-1}$ with a disappearance of ketone $\mathrm{C}=\mathrm{O}$ stretching vibration,which clearly confirmed that a formation of these compounds had been take place (Scheme II).

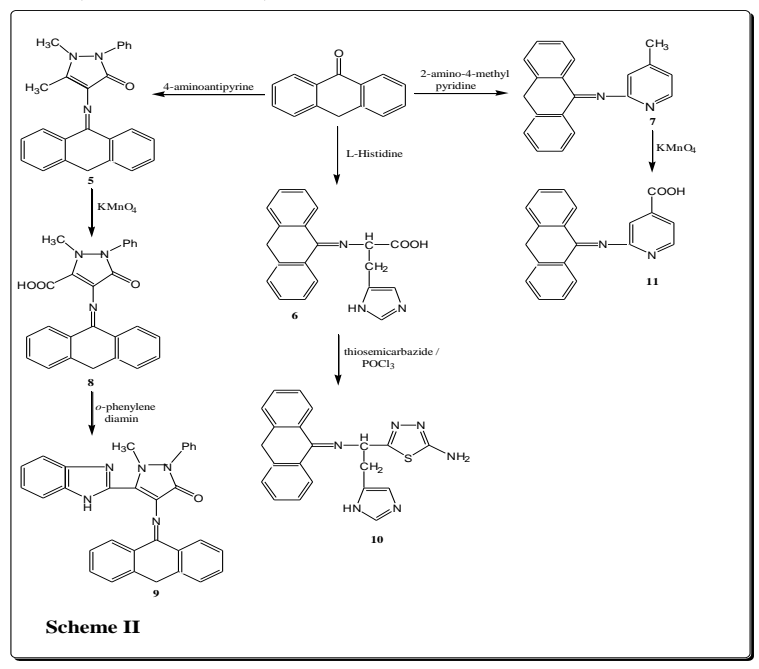

The $-\mathrm{CH}_{3}$ group of compounds 5 and 7 was oxidized by $\mathrm{KMnO}_{4}$ to afford 4- (anthracen -9 (10H)ylideneamino)-1-methyl-3-oxo-2phenyl-2,3-dihydro-1H-pyrazole -5carboxylic acid (8) and 2-(anthracen9(10H)-ylideneamino)-4-carboxylic acidpyridine (11), respectively, Scheme II. The IR spectra of compounds $\mathbf{8}$ and $\mathbf{1 1}$ showed characteristic carboxylic acid $\mathrm{C}=\mathrm{O}$ band at $1705 \mathrm{~cm}^{-1}$. ${ }^{1} \mathrm{H}-\mathrm{NMR}$ spectra showed characteristic bands at $\delta 9.89$, 9.92ppm, respectively, due $-\mathrm{OH}$ group, both disappeared with $\mathrm{D}_{2} \mathrm{O}$ exchange. Compounds $\mathbf{8}$ and $\mathbf{1 1}$ display, in addition to other signals, signal at 168.8 and $173.1 \mathrm{ppm}$ due to $\mathrm{C}=\mathrm{O}$ of carboxylic acid group.

Next, compound 8 condensed with $o$-phenylene diamine to give compounds 9. The structures of the new compound were assigned on the basis of their spectral data. ${ }^{13} \mathrm{C}-\mathrm{NMR}$ spectrum of compound 9 showed characteristic signal at $173.0 \mathrm{ppm}$ belonging to $\mathrm{C}=\mathrm{O}$ of pyrazolone ring. On the other hand, ${ }^{1} \mathrm{H}-\mathrm{NMR}$ spectrum of compound $\mathbf{9}$ displayed doublet at 8.79due to $-\mathrm{NH}$ group of benzimidazole ring which further assigned by $\mathrm{D}_{2} \mathrm{O}$ exchange. Compound 10 was obtained by the reaction of compound 6 with thiosemicarbazide, Scheme II. In the IR spectrum of this compound, the stretching band derived from $-\mathrm{OH}$ and $\mathrm{C}=\mathrm{O}$ of carboxylic acid was absent. In addition, signal derived from $\mathrm{NH}_{2}$ of 1,3,4-thiadiazole ring, at8.89, was observed which was disappeared by $\mathrm{D}_{2} \mathrm{O}$ exchange.

In the last part of this work, compound 17 was synthesized according to Scheme III.

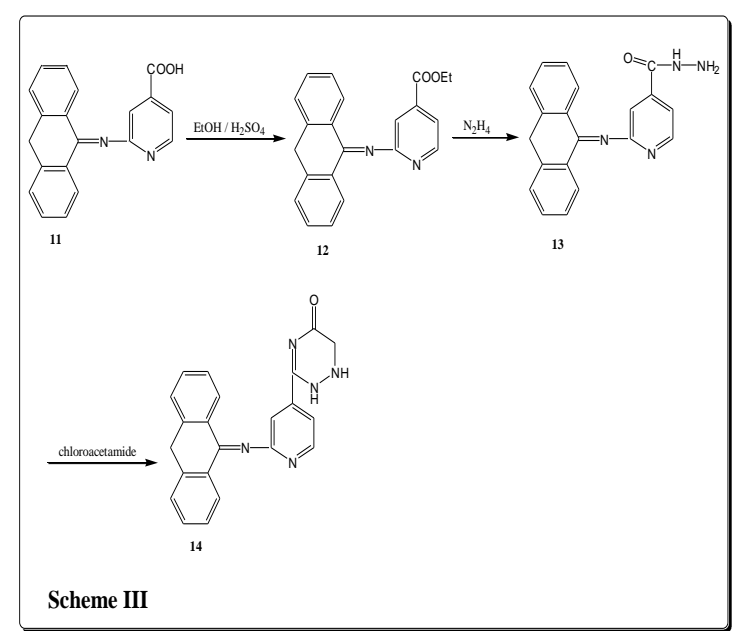


The IR spectra of compounds 12 and 13 showed stretching vibration of $\mathrm{C}=\mathrm{O}$ of ester and amide group at 1698 and $1645 \mathrm{~cm}^{-1}$, respectively. Compounds 12 display in its ${ }^{1} \mathrm{H}-\mathrm{NMR}$ spectrum, in addition to other signals, triplet at $\delta 1.68$ and quartet at $1.78 \mathrm{ppm}$ due to- $\mathrm{CH}_{2} \mathrm{CH}_{3}$ group, while ${ }^{1} \mathrm{H}-\mathrm{NMR}$ spectrum of compound $\mathbf{1 3}$ showed two singlet at 8.11 and $8.89 \mathrm{ppm}$ due to $\mathrm{NHNH}_{2}$ group which further characterized by $\mathrm{D}_{2} \mathrm{O}$ exchange. Treatment of compound $\mathbf{1 3}$ with chloroacetamide produced compound 17. The structure compound $\mathbf{1 4}$ was determined on the basis of its spectral and analytical data. ${ }^{1} \mathrm{H}-\mathrm{NMR}$ spectrum showed disappearance of signal at $8.89 \mathrm{ppm}$ due to $-\mathrm{NH}_{2}$ group of acid hydrazide with the appearance of two singlets at $\delta 7.98$ and $8.04 \mathrm{ppm}$ for two $-\mathrm{NH}$ groups of triazine ring, these two signals were disappeared with $\mathrm{D}_{2} \mathrm{O}$ exchange. Furthermore, ${ }^{13} \mathrm{C}-\mathrm{NMR}$ spectra of compounds 12-14 are in agreement with their IR and ${ }^{1} \mathrm{H}-\mathrm{NMR}$ data. Table 2 summarized the physical properties of synthesized compounds and Table 3 summarized the spectral data of synthesized compounds.

\section{Antimicrobial Activity}

All new synthesized compounds 1-14 were evaluated for antimicrobial activity against one strain of Gram +ve bacteria (Staphylococcus aureus), Gram -ve bacteria (Escherichia coli) and a fungus (Candida albicans) (Table 1) $[22]$.
Table 1: Antimicrobial activity for compounds (1-14)

\begin{tabular}{|c|c|c|c||}
\hline \multirow{2}{*}{$\begin{array}{c}\text { Compounds } \\
\text { No. }\end{array}$} & \multicolumn{3}{|c|}{ Micro-organism } \\
\cline { 2 - 4 } & Staphylococc & Escheric & Candid \\
& aureus & hia coli & albican \\
& & & $s$ \\
\hline \hline 1 & ++ & + & ++ \\
\hline 2 & ++ & ++ & ++ \\
\hline 3 & +++ & +++ & +++ \\
\hline 4 & +++ & +++ & +++ \\
\hline 5 & ++ & ++ & ++ \\
\hline 6 & ++ & + & + \\
\hline 7 & + & + & + \\
\hline 8 & ++ & ++ & ++ \\
\hline 9 & +++ & +++ & +++ \\
\hline 10 & +++ & +++ & +++ \\
\hline 11 & +++ & +++ & +++ \\
\hline 12 & + & + & + \\
\hline 13 & +++ & +++ & +++ \\
\hline 14 & ++ & +++ & +++ \\
\hline Streptomyci & +++ & +++ & +++ \\
\hline $\mathbf{n}$ & & & \\
\hline \hline
\end{tabular}

$$
\begin{array}{cc}
+ & =(5-10) \mathrm{mm}=\text { slightly active } \\
++ & =(11-20) \mathrm{mm}=\text { moderately active } \\
+++ & =\text { more than } 20 \mathrm{~mm}=\text { highly active }
\end{array}
$$

In general, all synthesized compounds exhibited good inhibitory activity against tested pathogenic microorganism. Compounds 3, 4, 9, 10, 11, 13 and 14 showed higher activity than the others. 
Table 2: Physical properties of compounds (1-14)

\begin{tabular}{|c|c|c|c|c|c||}
\hline $\begin{array}{c}\text { Comp. } \\
\text { No. }\end{array}$ & $\begin{array}{c}\text { M.P } \\
\left({ }^{\circ} \mathbf{C}\right)\end{array}$ & $\begin{array}{c}\text { Molecular } \\
\text { Formula }\end{array}$ & $\begin{array}{c}\text { Yield } \\
(\boldsymbol{\%})\end{array}$ & Color & Recrystalization solvent \\
\hline \hline 1 & $180-182$ & $\mathrm{C}_{20} \mathrm{H}_{16} \mathrm{~N}_{2}$ & 70 & Yellow & Chloroform \\
\hline 2 & $210-212$ & $\mathrm{C}_{15} \mathrm{H}_{13} \mathrm{~N}_{3} \mathrm{~S}$ & 75 & Yellow & Ethanol \\
\hline 3 & $180-182$ dec. & $\mathrm{C}_{16} \mathrm{H}_{17} \mathrm{~N}_{3} \mathrm{OS}$ & 75 & White & Acetone \\
\hline 4 & $60-62$ & $\mathrm{C}_{23} \mathrm{H}_{14} \mathrm{~N}_{3} \mathrm{SBr}$ & 66 & White & Dichloromethane \\
\hline 5 & $118-120$ & $\mathrm{C}_{23} \mathrm{H}_{19} \mathrm{~N}_{3} \mathrm{O}$ & 70 & Pale Yellow & Ethanol \\
\hline 6 & $140-142$ & $\mathrm{C}_{20} \mathrm{H}_{17} \mathrm{~N}_{3} \mathrm{O}_{2}$ & 70 & Yellow & Ethanol \\
\hline 7 & $92-94$ & $\mathrm{C}_{20} \mathrm{H}_{16} \mathrm{~N}_{2}$ & 57 & Brown & Ethanol \\
\hline 8 & $193-195$ & $\mathrm{C}_{23} \mathrm{H}_{17} \mathrm{~N}_{3} \mathrm{O}_{3}$ & 60 & White & Chloroform \\
\hline 9 & $120-122$ & $\mathrm{C}_{31} \mathrm{H}_{23} \mathrm{~N}_{5} \mathrm{O}$ & 72 & Milky & Ethanol \\
\hline 10 & $170-172$ dec. & $\mathrm{C}_{21} \mathrm{H}_{18} \mathrm{~N}_{6} \mathrm{~S}$ & 77 & Brown & Ethanol \\
\hline 11 & $126-128$ & $\mathrm{C}_{20} \mathrm{H}_{14} \mathrm{~N}_{2} \mathrm{O}_{2}$ & 60 & Gray & Ethanol \\
\hline 12 & $102-104$ & $\mathrm{C}_{22} \mathrm{H}_{18} \mathrm{~N}_{2} \mathrm{O}$ & 55 & Yellowish- & Green \\
\hline 13 & $188-190$ & $\mathrm{C}_{20} \mathrm{H}_{16} \mathrm{~N}_{4} \mathrm{O}$ & 75 & Light Yellow & - \\
\hline 14 & $89-91$ & $\mathrm{C}_{22} \mathrm{H}_{17} \mathrm{~N}_{5} \mathrm{O}$ & 60 & White & Chloroform \\
\hline \hline
\end{tabular}

Table 3: Spectral data of compounds (1-14)

\begin{tabular}{|c|c|c|c|c|}
\hline $\begin{array}{c}\text { Comp. } \\
\text { No. }\end{array}$ & FT-IR & ${ }^{1} \mathrm{H}-\mathrm{NMR}$ & ${ }^{13} \mathrm{C}-\mathrm{NMR}$ & CHN \\
\hline 1 & $\begin{array}{c}3200(v \mathrm{NH}), 3080(v \mathrm{CH} \\
\text { aromatic }) 2800(v \mathrm{CH} \\
\text { aliphatic }), 1550(v \mathrm{C}=\mathrm{C}), \\
755(\text { aromatic } o- \\
\text { substituted })\end{array}$ & $\begin{array}{c}2.02\left(\mathrm{~s}, 2 \mathrm{H}, \mathrm{CH}_{2}\right), 6.54- \\
7.73(\mathrm{~m}, 12 \mathrm{H}, \mathrm{Ar}-\mathrm{H}) \\
8.62,8.73(2 \mathrm{~s}, 2 \mathrm{H}, \\
2 \mathrm{NH})\left(\mathrm{D}_{2} \mathrm{O} \text { exchange, }\right. \\
\text { disappear })\end{array}$ & $\begin{array}{c}20.2\left(1 \mathrm{C}, \mathrm{CH}_{2}\right), \\
26.2(1 \mathrm{C}, \mathrm{N}-\mathrm{C}-\mathrm{N}), \\
125.0-140.7(18 \mathrm{C}, \\
\text { aromatic carbons })\end{array}$ & $\begin{array}{l}\text { Anal. Calcd: C, } \\
\text { 83.33; H, 5.56; } \\
\text { N,11.11. Found: } \\
\text { C,83.67; H,5.23; } \\
\text { N,10.98 }\end{array}$ \\
\hline 2 & $\begin{array}{c}3330-3200(v \text { asym, sym } \\
\left.\mathrm{NH}_{2}, \mathrm{NH}\right), 3100(v \mathrm{CH} \\
\text { aromatic }), 2880(v \mathrm{CH} \\
\text { aliphatic }), 1620(v \mathrm{C}=\mathrm{N}), \\
1550(v \mathrm{C}=\mathrm{C}), 1330 \\
(v \mathrm{C}=\mathrm{S}), 765 \text { (aromatic } o- \\
\text { substituted) }\end{array}$ & $\begin{array}{l}1.87\left(\mathrm{~s}, 2 \mathrm{H}, \mathrm{CH}_{2}\right), 7.34- \\
7.77(\mathrm{~m}, 8 \mathrm{H}, \mathrm{Ar}-\underline{\mathrm{H}}), \\
8.53(\mathrm{~s}, 1 \mathrm{H}, \mathrm{NH})\left(\mathrm{D}_{2} \mathrm{O}\right. \\
\text { exchange, disappear }), \\
9.32\left(\mathrm{~s}, 2 \mathrm{H}, \mathrm{NH}_{2}\right)\left(\mathrm{D}_{2} \mathrm{O}\right. \\
\text { exchange, disappear }) .\end{array}$ & $\begin{array}{c}{ }^{3} \mathrm{C}-\mathrm{NMR} \delta: 22.2(1 \mathrm{C}, \\
\left.\mathrm{CH}_{2}\right), 96.9(1 \mathrm{C}, \underline{\mathrm{C}}=\mathrm{N}), \\
100.2\left(1 \mathrm{C}, \mathrm{S}=\mathbf{C}-\mathrm{NH}_{2}\right), \\
128.0-136.7(12 \mathrm{C}, \\
\text { aromatic carbons })\end{array}$ & $\begin{array}{c}\text { Anal. Calcd: C, } \\
\text { 67.42; H, 4.87; N, } \\
\text { 15.73; S, } \\
\text { 11.99.Found: } \\
\text { C,67.46; H,4.72; } \\
\text { N,15.42; S,11.75 }\end{array}$ \\
\hline 3 & $\begin{array}{c}3200(v \mathrm{NH}), 3080(v \mathrm{CH} \\
\text { aromatic }), 2900(v \mathrm{CH} \\
\text { aliphatic }), 1685(v \mathrm{C}=\mathrm{O}), \\
1630(v \mathrm{C}=\mathrm{N}), 1550 \\
(v \mathrm{C}=\mathrm{C}), 760(\text { aromatic } o- \\
\text { substituted }), 662(\mathrm{C}-\mathrm{S})\end{array}$ & $\begin{array}{c}2.32\left(\mathrm{~s}, 2 \mathrm{H}, \mathrm{CH}_{2}\right) \\
2.65\left(\mathrm{~s}, 2 \mathrm{H}, \mathrm{N}-\mathrm{CH}_{2}\right) \\
6.74-7.85(\mathrm{~m}, 8 \mathrm{H}, \mathrm{Ar}- \\
\underline{\mathbf{H}}), 8.51(\mathrm{~s}, 1 \mathrm{H}, \mathrm{NH}) \\
\left(\mathrm{D}_{2} \mathrm{O} \text { exchange, }\right. \\
\text { disappear })\end{array}$ & $\begin{array}{c}20.1\left(1 \mathrm{C}, \mathrm{CH}_{2}\right), \\
26.3\left(1 \mathrm{C}, \underline{\mathrm{C}} \mathrm{H}_{2} \text { adjacent }\right. \\
\text { to carbonyl }), 92.3, \\
94.0(2 \mathrm{C}, 2 \mathrm{C}=\mathrm{N}), \\
128.8-133.0(12 \mathrm{C}, \\
\text { aromatic carbons }), \\
173.2(1 \mathrm{C}, \mathrm{C}=\mathrm{O})\end{array}$ & $\begin{array}{l}\text { Anal. Calcd: C, } \\
66.45 ; \mathrm{H}, 4.23 ; \mathrm{N}, \\
\text { 13.68; S, 10.42. } \\
\text { Found: C,66.30; } \\
\text { H,4.30; N,15.54; } \\
\text { S,10.35 }\end{array}$ \\
\hline 4 & $\begin{array}{c}3270(v \mathrm{NH}), 3033(v \mathrm{CH} \\
\text { aromatic }), 2855(v \mathrm{CH} \\
\text { aliphatic), } 1625(v \mathrm{C}=\mathrm{N}), \\
1545(v \mathrm{C}=\mathrm{C}), 847 \\
\text { (aromatic } p \text {-substituted), } \\
735(\text { aromatic } o- \\
\text { substituted), } 650(\mathrm{C}-\mathrm{S})\end{array}$ & $\begin{array}{c}\left.\text { 2.14(s, 2H, } \mathrm{CH}_{2}\right), \\
\text { 5.67(1H, S-CH}=), \\
6.41-7.78(\mathrm{~m}, 12 \mathrm{H}, \mathrm{Ar}- \\
\underline{\mathbf{H}}), 8.81(\mathrm{~s}, 1 \mathrm{H}, \mathrm{NH}) \\
\left(\mathrm{D}_{2} \mathrm{O} \text { exchange, }\right. \\
\text { disappear) }\end{array}$ & $\begin{array}{c}23.4\left(1 \mathrm{C}, \mathrm{CH}_{2}\right), 87.8, \\
89.3(2 \mathrm{C}, \underline{\mathbf{C}}=\underline{\mathbf{C}}), 100.2, \\
100.7(2 \mathrm{C}, \underline{\mathbf{C}}=\mathrm{N}), \\
133.5-138.1(18 \mathrm{C}, \\
\text { aromatic carbons })\end{array}$ & $\begin{array}{c}\text { Anal. Calcd: C, } \\
\text { 62.16; H, 3.15; N, } \\
\text { 9.46; S, 7.21. Found: } \\
\text { C,62.02; H,2.89; } \\
\text { N,9.13; S,7.33 }\end{array}$ \\
\hline
\end{tabular}

\begin{tabular}{||l|l|l|l|c||}
\hline \hline Comp. & FT-IR & ${ }^{1}$ H-NMR & ${ }^{13}$ C-NMR & CHN \\
\hline
\end{tabular}




\begin{tabular}{|c|c|c|c|c|}
\hline No. & & & & \\
\hline 5 & $\begin{array}{c}3080(v \mathrm{CH} \text { aromatic }), \\
2860(v \mathrm{CH} \text { aliphatic }), \\
1683(v \mathrm{C}=\mathrm{O}), 1625 \\
(v \mathrm{C}=\mathrm{N}), 1580(v \mathrm{C}=\mathrm{C})\end{array}$ & $\begin{array}{c}1.56,1.81\left(2 \mathrm{~s}, 6 \mathrm{H}, 2 \mathrm{CH}_{3}\right) \\
2.21\left(\mathrm{~s}, 2 \mathrm{H}, \mathrm{CH}_{2}\right), 6.04- \\
7.11(\mathrm{~m}, 13 \mathrm{H}, \mathrm{Ar}-\underline{\mathbf{H}})\end{array}$ & $\begin{array}{c}17.2,17.8\left(2 \mathrm{C}, 2 \mathrm{CH}_{3}\right), \\
22.5\left(1 \mathrm{C}, \mathrm{CH}_{2}\right), 92.0(1 \mathrm{C}, \underline{\mathbf{C}}- \\
\left.\mathrm{CH}_{3}\right), 93.4(1 \mathrm{C},-\mathrm{N}- \\
\left.\underline{\mathrm{C}}=\mathrm{C}\left(\mathrm{CH}_{3}\right)\right), 95.8(1 \mathrm{C},-\underline{\mathbf{C}}=\mathrm{N}- \\
), 125.6-135.2(18 \mathrm{C}, \\
\text { aromatic carbons }), 170.1(1 \mathrm{C}, \\
\mathrm{C}=\mathrm{O})\end{array}$ & $\begin{array}{c}\text { Anal. Calcd: } \\
\text { C, 78.19; H, } \\
\text { 5.38; N, } \\
11.90 \\
\text { Found: C, } \\
\text { 77.90; H, } \\
\text { 5.12; N, } \\
11.59 \\
\end{array}$ \\
\hline 6 & $\begin{array}{c}3400-3300(\mathrm{~b}, v \mathrm{OH}), \\
3088(v \mathrm{CH} \text { aromatic }), \\
2830(v \mathrm{CH} \text { aliphatic }), \\
1705(v \mathrm{C}=\mathrm{O}), 1620 \\
(v \mathrm{C}=\mathrm{N})\end{array}$ & $\begin{array}{c}2.05\left(\mathrm{~s}, 2 \mathrm{H}, \mathrm{CH}_{2}\right), 2.40(\mathrm{~d}, \\
\left.2 \mathrm{H}, \mathrm{CH}_{2}\right), 2.87(\mathrm{t}, 1 \mathrm{H}, \\
\mathrm{CH}), 5.27,5.63(2 \mathrm{~s}, 2 \mathrm{H}, \\
2=\mathrm{C} \underline{\mathbf{H}}), 7.05-7.76(\mathrm{~m}, 8 \mathrm{H}, \\
\mathrm{Ar}-\underline{\mathbf{H}}), 8.58(\mathrm{~s}, 1 \mathrm{H}, \mathrm{NH}) \\
\left(\mathrm{D}_{2} \mathrm{O} \text { exchange, }\right. \\
\text { disappear }), 9.49(\mathrm{~s}, 1 \mathrm{H}, \\
\mathrm{OH})\left(\mathrm{D}_{2} \mathrm{O} \text { exchange, }\right. \\
\text { disappear) }\end{array}$ & $\begin{array}{c}16.8,17.4\left(1 \mathrm{C}, \mathrm{CH}_{2}\right), \\
22.0(1 \mathrm{C},-\mathbf{C} H-), 102.1(1 \mathrm{C},- \\
\mathbf{C}=\mathrm{N}), 118.5,120.3,122.0 \\
\text { (imidazole carbons), 127.3- } \\
133.9(12 \mathrm{C}, \text { aromatic } \\
\text { carbons }), 171.7(1 \mathrm{C}, \mathrm{C}=\mathrm{O})\end{array}$ & $\begin{array}{c}\text { Anal. Calcd: } \\
\text { C, 72.51; H, } \\
\text { 5.14; N, } \\
\text { 12.69. } \\
\text { Found: C, } \\
\text { 72.62; H, } \\
\text { 5.05; N, } \\
12.47\end{array}$ \\
\hline 7 & $\begin{array}{c}3100(v \mathrm{CH} \\
\text { aromatic }), 2855(v \mathrm{CH} \\
\text { aliphatic }), 1625(v \mathrm{C}=\mathrm{N})\end{array}$ & 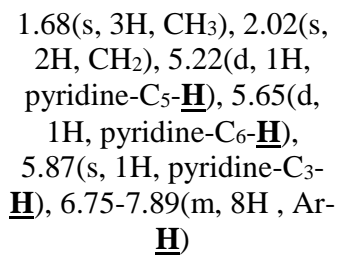 & $\begin{array}{c}17.5\left(1 \mathrm{C}, \mathrm{CH}_{3}\right), 20.2(1 \mathrm{C}, \\
\left.\mathrm{CH}_{2}\right), 94.1,95.7,96.3,98.8 \\
100.1 \text { (pyridine ring } \\
\text { carbons), } 103.3(1 \mathrm{C},-\mathbf{C}=\mathrm{N}- \\
), 127.0-134.5(12 \mathrm{C}, \\
\text { aromatic carbons) }\end{array}$ & $\begin{array}{c}\text { Anal. Calcd: } \\
\text { C, } 84.51 ; \mathrm{H}, \\
\text { 5.63; N, } 9.86 . \\
\text { Found: C, } \\
84.32 ; \mathrm{H}, \\
\text { 5.70; N, } 9.76 .\end{array}$ \\
\hline 8 & $\begin{array}{c}3400-3320(\mathrm{~b}, v \mathrm{OH}), 3089 \\
(v \mathrm{CH} \text { aromatic }), 2850 \\
(v \mathrm{CH} \text { aliphatic }), 1705 \\
1685(v \mathrm{C}=\mathrm{O}), 1620(v \mathrm{C}=\mathrm{N})\end{array}$ & $\begin{array}{c}1.88\left(\mathrm{~s}, 3 \mathrm{H}, \mathrm{CH}_{3}\right), 2.76(\mathrm{~s}, \\
\left.2 \mathrm{H}, \mathrm{CH}_{2}\right), 6.55-7.98(\mathrm{~m}, \\
13 \mathrm{H}, \mathrm{Ar}-\underline{\mathbf{H}}), 9.89(\mathrm{~s}, 1 \mathrm{H}, \\
\mathrm{OH})\left(\overline{\mathrm{D}_{2} \mathrm{O} \text { exchange, }}\right. \\
\text { disappear })\end{array}$ & $\begin{array}{c}16.1\left(1 \mathrm{C}, \mathrm{CH}_{3}\right), 18.2(1 \mathrm{C} \\
\left.\mathrm{CH}_{2}\right), 110.6,113.1(2 \mathrm{C} \\
\underline{\mathbf{C}}=\underline{\mathbf{C}}), 115.4(1 \mathrm{C}, \underline{\mathbf{C}}=\mathrm{N}) \\
130.5-137.4(18 \mathrm{C}, \text { aromatic } \\
\text { carbons }), 168.8(\mathrm{C}=\mathrm{O})\end{array}$ & $\begin{array}{c}\text { Anal. Calcd: } \\
\text { C, 73.35; H, } \\
\text { 4.65; N, } \\
\text { 10.27. } \\
\text { Found: C, } \\
\text { 73.11; H, } \\
\text { 4.45; N, } \\
10.32 \\
\end{array}$ \\
\hline 9 & $\begin{array}{c}3200(v \mathrm{NH}), 3050(v \mathrm{CH} \\
\text { aromatic }), 2975(v \mathrm{CH} \\
\text { aliphatic }), 1670(v \mathrm{C}=\mathrm{O})\end{array}$ & $\begin{array}{c}1.82\left(\mathrm{~s}, 3 \mathrm{H}, \mathrm{CH}_{3}\right), 2.51(\mathrm{~s}, \\
\left.2 \mathrm{H}, \mathrm{CH}_{2}\right), 6.47-8.00(\mathrm{~m}, \\
17 \mathrm{H}, \mathrm{Ar}-\mathrm{H}), 8.79(\mathrm{~s}, 1 \mathrm{H}, \\
\mathrm{NH})\left(\mathrm{D}_{2} \mathrm{O} \text { exchange, }\right. \\
\text { disappear })\end{array}$ & $\begin{array}{c}17.1\left(1 \mathrm{C}, \mathrm{CH}_{3}\right), 18.4(1 \mathrm{C}, \\
\left.\mathrm{CH}_{2}\right), 111.3,113.4(2 \mathrm{C}, \\
\mathbf{C}=\mathrm{C}), 115.5,117.0(2 \mathrm{C}, \\
\mathbf{C}=\mathrm{N}), 128.4-135.7(24 \mathrm{C}, \\
\text { aromatic carbons }), 173.0 \\
(1 \mathrm{C}, \mathrm{C}=\mathrm{O})\end{array}$ & $\begin{array}{c}\text { Anal. Calcd: } \\
\text { C, 77.34; H, } \\
\text { 4.78; N, } \\
\text { 14.55. } \\
\text { Found: C, } \\
\text { 77.12; H, } \\
\text { 4.54; N, } \\
14.33 \\
\end{array}$ \\
\hline 10 & $\begin{array}{l}3300-3180(v \text { asym, sym } \\
\left.\mathrm{NH}, \mathrm{NH}_{2}\right), 3090(v \mathrm{CH} \\
\text { aromatic }), 2900(v \mathrm{CH} \\
\text { aliphatic }), 1625(v \mathrm{C}=\mathrm{N})\end{array}$ & $\begin{array}{c}1.98\left(\mathrm{~s}, 2 \mathrm{H}, \mathrm{CH}_{2}\right), 2.15(\mathrm{~d}, \\
\left.2 \mathrm{H}, \mathrm{CH}_{2}\right), 2.67(\mathrm{t}, 1 \mathrm{H}, \\
\mathrm{CH}), 5.38,5.77(2 \mathrm{~s}, 2 \mathrm{H}, \\
=\mathrm{CH}), 6.48-7.77(\mathrm{~m}, 8 \mathrm{H}, \\
\mathrm{Ar}-\mathrm{H} \text { and imidazole-H }), \\
8.15(\mathrm{~s}, 1 \mathrm{H}, \mathrm{NH})\left(\mathrm{D}_{2} \mathrm{O}\right. \\
\text { exchange, disappear }) \\
\left.\text { 8.89(s, } 2 \mathrm{H}, \mathrm{NH}_{2}\right)\left(\mathrm{D}_{2} \mathrm{O}\right. \\
\text { exchange, disappear })\end{array}$ & $\begin{array}{c}17.2,17.8\left(2 \mathrm{C}, 2 \mathrm{CH}_{2}\right), \\
21.3(1 \mathrm{C},-\mathrm{CH}-), 105.0(1 \mathrm{C},- \\
\mathbf{C}=\mathrm{N}-), 120.2,121.4, \\
123.5(\text { imidazole carbons), } \\
\text { 129.0-135.5(12C, aromatic } \\
\text { carbons), 139.4, } \\
\text { 140.5(thiadiazole carbons) }\end{array}$ & $\begin{array}{c}\text { Anal. Calcd: } \\
\text { C, 65.28; H, } \\
\text { 4.66; N, } \\
\text { 21.76, S, } \\
\text { 8.29. Found: } \\
\text { C, 65.10; H, } \\
\text { 4.73; N, } \\
\text { 21.54; S, } \\
8.36\end{array}$ \\
\hline 11 & $\begin{array}{c}3376(\mathrm{~b}, v \mathrm{OH}), 3100(v \mathrm{CH} \\
\text { aromatic }), 2950(v \mathrm{CH} \\
\text { aliphatic), } 1620(v \mathrm{C}=\mathrm{N}), \\
1705(v \mathrm{C}=\mathrm{O})\end{array}$ & $\begin{array}{c}2.02\left(\mathrm{~s}, 2 \mathrm{H}, \mathrm{CH}_{2}\right), 6.14(\mathrm{~s}, \\
1 \mathrm{H},=\mathrm{CH}-), 6.32-6.36(\mathrm{~d}, \\
1 \mathrm{H},=\mathrm{CH}), 6.40-6.45(\mathrm{~d} \\
1 \mathrm{H},=\mathrm{CH}), 6.87-7.15(\mathrm{~m}, \\
8 \mathrm{H}, \mathrm{Ar}-\mathrm{H}), 9.92(\mathrm{~s}, 1 \mathrm{H} \\
\mathrm{OH})\left(\mathrm{D}_{2} \mathrm{O} \text { exchange }\right. \\
\text { disappear })\end{array}$ & $\begin{array}{c}17.6\left(1 \mathrm{C}, \mathrm{CH}_{2}\right), 106.4(1 \mathrm{C}, \\
\mathrm{C}=\mathrm{N}), 110.7,112.3,114.1, \\
115.0,117.5 \text { (pyridine } \\
\text { carbons), 128.3-133.6(12C, } \\
\text { aromatic carbons), 173.7(1C, } \\
\mathrm{C}=\mathrm{O})\end{array}$ & $\begin{array}{c}\text { Anal. Calcd: } \\
\text { C, } 76.43 ; \mathrm{H}, \\
\text { 4.46; N, } 8.92 . \\
\text { Found: C, } \\
76.22 ; \mathrm{H}, \\
4.52 ; \mathrm{N}, 8.85\end{array}$ \\
\hline
\end{tabular}




\begin{tabular}{|c|c|c|c|c|}
\hline $\begin{array}{c}\text { Comp. } \\
\text { No. }\end{array}$ & FT-IR & ${ }^{1} \mathrm{H}-\mathrm{NMR}$ & ${ }^{13} \mathrm{C}-\mathrm{NMR}$ & CHN \\
\hline 12 & $\begin{array}{c}3078(v \mathrm{CH} \text { aromatic }), \\
2823(v \mathrm{CH} \text { aliphatic }), \\
1620(v \mathrm{C}=\mathrm{N}), 1698 \\
(v \mathrm{C}=\mathrm{O})\end{array}$ & $\begin{array}{c}1.68\left(\mathrm{t}, 3 \mathrm{H}, \mathrm{CH}_{3}\right), 1.78(\mathrm{q}, 2 \mathrm{H}, \\
\left.\mathrm{CH}_{2}\right), 2.05\left(\mathrm{~s}, 2 \mathrm{H}, \mathrm{CH}_{2}\right) \\
6.15(\mathrm{~s}, 1 \mathrm{H},=\mathrm{CH}-), 6.30- \\
6.35(\mathrm{~d}, 1 \mathrm{H},=\mathrm{CH}), 6.42- \\
6.67(\mathrm{~d}, 1 \mathrm{H},=\mathrm{CH}), 7.03- \\
7.95(\mathrm{~m}, 8 \mathrm{H}, \mathrm{Ar}-\mathbf{H})\end{array}$ & $\begin{array}{c}15.5\left(1 \mathrm{C}, \mathrm{CH}_{2}\right), 16.7,18.3(2 \mathrm{C}, \\
\left.-\mathrm{CH}_{2} \mathrm{CH}_{3}\right), 105.5(1 \mathrm{C}, \mathbf{C}=\mathrm{N}), \\
112.4,113.2,115.7,118.0, \\
121.1(5 \mathrm{C}, \text { pyridine carbons }), \\
112.4-121.1(6 \mathrm{C}, \text { olefinic } \\
\text { carbons }), 130.3-134.8(12 \mathrm{C}, \\
\text { aromatic carbons }), 169.1(1 \mathrm{C}, \\
\mathrm{C}=\mathrm{O})\end{array}$ & $\begin{array}{l}\text { Anal. Calcd: } \\
\text { C, 80.98; H, } \\
\text { 5.52; N, 8.59 } \\
\text { Found: C, } \\
80.70 ; \mathrm{H} \text {, } \\
\text { 5.67; N, } 8.44\end{array}$ \\
\hline 13 & $\begin{array}{c}3300-3200(v \text { asym, } \\
\left.\text { sym }-\mathrm{NHNH}_{2}\right), \\
3089(v \mathrm{CH} \text { aromatic }), \\
2967(v \mathrm{CH} \text { aliphatic }), \\
1630(v \mathrm{C}=\mathrm{N}), 1645 \\
(v \mathrm{C}=\mathrm{O})\end{array}$ & $\begin{array}{c}2.02\left(\mathrm{~s}, 2 \mathrm{H}, \mathrm{CH}_{2}\right), 5.98(\mathrm{~s}, 1 \mathrm{H}, \\
=\mathrm{CH}-), 6.43-6.55(\mathrm{~d}, 1 \mathrm{H}, \\
=\mathrm{CH}), 6.57-6.63(\mathrm{~d}, 1 \mathrm{H}, \\
=\mathrm{CH}), 7.07-7.77(\mathrm{~m}, 8 \mathrm{H}, \mathrm{Ar}- \\
\mathbf{H}), 8.11(\mathrm{~s}, 1 \mathrm{H}, \mathrm{NH})\left(\mathrm{D}_{2} \mathrm{O}\right. \\
\text { exchange, disappear }), 8.89(\mathrm{~s}, \\
\left.2 \mathrm{H}, \mathrm{NH}_{2}\right)\left(\mathrm{D}_{2} \mathrm{O} \text { exchange, }\right. \\
\text { disappear })\end{array}$ & $\begin{array}{c}.22\left(1 \mathrm{C}, \mathrm{CH}_{2}\right), 107.3(1 \mathrm{C}, \\
\mathrm{C}=\mathrm{N}), 110.7,113.4,116.0, \\
117.8,120.1(5 \mathrm{C}, \text { pyridine } \\
\text { carbons }), 110.7-120.1(6 \mathrm{C}, \\
\text { olefinic carbons }), 130.3- \\
137.4(12 \mathrm{C}, \text { aromatic } \\
\text { carbons }), 168.2(1 \mathrm{C}, \mathrm{C}=\mathrm{O})\end{array}$ & $\begin{array}{c}\text { Anal. Calcd: } \\
\text { C, 73.17; H, } \\
\text { 4.88; N, } \\
\text { 17.07. } \\
\text { Found: C, } \\
\text { 73.00; H, } \\
\text { 4.73; N, } \\
\text { 16.80 } \\
\end{array}$ \\
\hline 14 & $\begin{array}{c}3220-3200(v \text { asym, } \\
\text { sym } \mathrm{NH}), 3067(v \mathrm{CH} \\
\text { aromatic }), 2955(v \mathrm{CH} \\
\text { aliphatic }), 1665 \\
(v \mathrm{C}=\mathrm{O}), 1620(v \mathrm{C}=\mathrm{N})\end{array}$ & $\begin{array}{c}2.01\left(\mathrm{~s}, 2 \mathrm{H}, \mathrm{CH}_{2}\right), 2.55(\mathrm{~s}, 2 \mathrm{H}, \\
\left.\text { triazine } \mathrm{CH}_{2}\right), 6.06,6.71(\mathrm{~s}, \\
1 \mathrm{H},=\mathrm{CH}-), 6.06-6.33(1 \mathrm{H}, \mathrm{d}, \\
=\mathrm{CH}), 6.37-6.42(\mathrm{~d}, 1 \mathrm{H}, \\
=\mathrm{CH}), 7.11-7.68(\mathrm{~m}, 8 \mathrm{H}, \mathrm{Ar}- \\
\mathbf{H}), 7.98,8.04(2 \mathrm{~s}, 2 \mathrm{H}, 2 \mathrm{NH}) \\
\left(\mathrm{D}_{2} \mathrm{O} \text { exchange, disappear }\right)\end{array}$ & $\begin{array}{c}20.2,23.7\left(2 \mathrm{C}, 2 \mathrm{CH}_{2}\right), \\
106.3(1 \mathrm{C}, \mathbf{C}=\mathrm{N}), 112.0, \\
115.6,117.8,119.3, \\
121.5(5 \mathrm{C}, \text { pyridine carbons }), \\
122.4,124.0(2 \mathrm{C}, \text { triazine } \\
\text { carbons }), 126.6-134.3(12 \mathrm{C}, \\
\text { aromatic carbons }), 173.3(1 \mathrm{C}, \\
\mathrm{C}=\mathrm{O})\end{array}$ & - \\
\hline
\end{tabular}

\section{References:}

1. Jarrahpour, A., D. Khalili, E. D. Clercq, C. Salmi and J. Michel, 2007. Synthesis, antibacterial, antifungal and antiviral activity evaluation of some new bis-Schiff bases of isatin and their derivatives. Molecules. 12: 1720-1730.

2. Al-Sha'alan ,N. H., 2007. Antimicrobial activity and spectral, magnetic and thermal studies of some transition metal complexes of a Schiff base hydrazone containing a quinoline moiety. Molecules. 12: 1080-1091.

3. Toggi, A. E., A. M. Hafez, H. Wack, B. Young and D. Lectka, 2002. The development of the first catalyzed reaction of ketenes and imines: catalytic, asymmetric synthesis of $\beta$-Lactams. Am. Chem. Soc. 124: 6626-6635.

4. Rodriguez, M. L. L., B. Benhamu, M. J. Morcillo and M. I. Martin, 1999. Synthesis and structureactivity relationships of new azabicyclic benzimidazole-4carboxylic acid derivatives with affinity for serotoninergic $5-\mathrm{HT}_{3}$ receptors. J. Med. Chem. 42: 50205028.

5. Roth, T., M. L. Marningstar, P. L. Boyer, S. H. Hughes and C. J. Michejda, 1997. Synthesis of substituted benzimidazoles as inhibition agents of HIV reverse transcription and for the treatment of HIV infection. J. Med. Chem. 40: 4199-4207.

6. Coburn, R. A., M. T. Clark, R. T. Evans and R. J. Genco, 1987. Alkyloxyamino substituted benzimidazoles and their use as protein kinase-C inhibitors. J. Med. Chem. 30: 205-208.

7. Roth, T., M. L. Morningstar, P. L.and R. W. Buckheit, 1999. Substituted benzimidazoles and their uses for inhibition of HIV reverse transcription and for the treatment of HIV infection. J. Med. Chem. 34: 2199-2207. 
8. Chen, A. Y., C. Yu, A. Bodley and L. F. Liu, 1993. Microwaveassisted synthesis of benzimidazole and thiazolidinone derivatives as HIV-1 RT inhibitors. Cancer Research. 53: 1332-1337.

9. Evans, D., T. A. Hicks, W. R. N. Williamson and E. A. Kitchen, 1996. 2-(2,4-Dichlorophenyl)-5fluoro-6-morpholin-4-yl- $1 H$ -

benzimidazole monohydrate. Eur. J. Med. Chem. 31: 635-642.

10. Choi, Y. K., K. H. Chjo, S. M. Park and N. D. Doddapaneni, 1995. Synthesis of new substituted antipyrines: electrochemical and spectroscopic properties. J. Electrochem. Soc. 142: 41074113.

11. Katia , B., L. Simon, R. Anne, C. Gerard and M. Bernard, 2004. Synthesis, structural determination and antibacterial activity of compounds derived from vanillin and 4-aminoantipyrine. J. Serb. Chem. Soc. 69 (12): 991-998.

12. Hodnett, E. M. and P. D. Mooney, 1970. 2-Dicyanomethylene-5,6dimethyl-1,2-dihydropyrazole-3carbonitrile. J. Med. Chem. 13: 786-790.

13. Hodnett, E. M. and W. J. Dunn, 1972. The effect of aging on anaerobic and aerobic enzyme activities in human skeletal muscle. J. Med. Chem. 15: 339-344.

14. Sengupta, P. K., M. R. Ray and S. S. Chakravorti, 1978. Synthesis of some new fused 1,3,4-thiadiazole derivatives and study of their antimicrobial activity. Indian $\mathrm{J}$. Chem. 16: 231-235.

15. Uher, M. and D. Berkes, 1999. Synthesis of some new substituted 1,2,4-triazole and1,3,4-thiadiazole and their derivatives. Chem. Papers. 53(3): 215-220.

16. Palaska,E., G. Şahin, P. Kelicen and G. Altinok, 2002. Synthesis and anti-inflammatory activity of 1-acylthiosemicarbazides, 1,3,4oxadiazoles, 1,3,4-thiadiazoles and 1,2,4-triazole-3-thiones. Farmaco. 57: 101-107.

17. Andres, C. J., J. J. Bronson, M. S. Deshpande and A. W. Walsh, 2000. Anti-malarials activity of 1,3,4-thiadiazole compounds. Med. Chem. Lett. 10: 715-721.

18. Uçucu, Ü., N. Gündoğdu and I. Işikadağ, 2001. Synthesis and analgesic activity of some 1benzyl-2-substituted-4,5-diphenyl-

1H-imidazole derivatives. IL Farmaco. 56: 285-290.

19. Al-Azzawi, R. W., 2007. Evaluation of Some Properties of Three Types of Denture Reline Materials with Miconazole (Antifungal agent) Preparation. A master thesis, Prosthetic

Department, University of Baghdad.

20. Demirbaş, N., 2005. Synthesis and characterization of new triheterocyclic compounds consisting of 1,2,4-triazol-3one,1,3,4-thiadiazole and 1,3,4oxadiazole rings. Turk. J. Chem. 29: 125-133.

21. Silverstein, R. M. and Webster F. X. 1998. Spectroscopic Identification of Organic Compounds. $6^{\text {th }}$ Ed. John Wiely \& Sons, Inc., New York, pp.92.

22. Limban, C., M. C. B. Chifiriuc, A. V. Missir, I. C. Chiriţă and C. Bleotu, 2008. Antimicrobial activity of some new thioureides derived from 2-(4chlorophenoxymethyl)benzoic acid. Molecules 13: 567-580 


\title{
تخليق مركبات حلقية غير متجانسة جديدة مشتقة من الأنثرون
}

وتقيم فعاليتها البايولوجية

\author{
نادية عادل صالج
}

"ققم الكيمياء/ كلية العلوم/ جامعة النهرين/ بغداد/ العراق

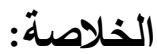

تم في هذا البحث تحضير قواعد شف مشتقة من تفاعل الانثرون مع امينات حلقية غير متجانسة.

المركبات الناتجة فوعلت مع نيوكليوفيلات مختلفة لانتاج نوع جديد من المركبات غير الحلقية. شخصت المواد

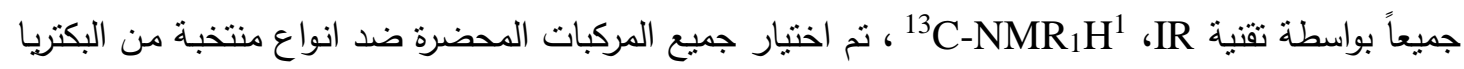

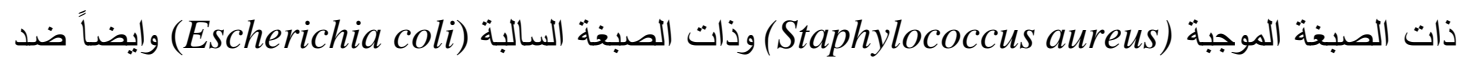

الفطريات. 